\title{
Why we need to be vigilant over myocardial thickening in oncology patients? A cardiac magnetic resonance case.
}

\author{
Cagdas Topel $^{1}$ and SAMET SEVINÇ ${ }^{1}$ \\ ${ }^{1}$ Mehmet Akif Ersoy Thoracic and Cardiovascular Research and Education Hospital
}

October 1,2020

\begin{abstract}
We present a patient who was on active surveillance for lung adenocarcinoma and hypertrophic septal cardiomyopathy (HCM), was referred for cardiac magnetic resonance (CMR) assessment for risk stratification due to HCM. CMR revealed a large myocardial metastasis obstructing mid-apical left and right ventricular cavity. Any wall thickening in oncology patients should prompt to perform further imaging before establishing certain diagnosis.
\end{abstract}

\section{CASE REPORT}

A 42-year-old male diagnosed with HCM previously, was referred our cardiac magnetic resonance (CMR) unit for evaluation of myocardial scarring and risk stratification by means of late gadolinium enhancement (LGE). His chief complain was increasing shortness of breath and was on active surveillance for prior lung adenocancer. ECG showed no significant abnormalities. Previous echocardiography exams showed hypertrophic septal cardiomyopathy with maximum wall thickness of $21 \mathrm{~mm}$. Current echocardiography exam showed mass-like thickening of the septal myocardium which nearly obscured the RV cavity (Figure 1, Movie 1). CMR showed significant wall thickening in the LV septum and apex. T1 and T2 weighted images showed that the thickened segment was isointense to normal myocardium (Figure 2, Movie 2). Mid to apical left ventricular and right ventricular cavity was obliterated due to its mass effect. First-pass perfusion study with gadolinium showed a large, mass-like appearance of the thickened myocardium and the mass had necrotic center (Movie 3). LGE images confirmed the large myocardial mass and its necrotic center (Figure 3). The findings were most consisted with myocardial metastasis.

\section{DISCUSSION}

Although primary tumor of the heart is rare with the reported prevalence of $0.1 \%-0.01$, metastatic cardiac tumor is more frequent than primary tumors with the range of $0.7 \%-3.5 \%$ in autopsy series. ${ }^{1}$ The prevalence of cardiac metastasis is reported up to $9.1 \%$ in oncology patients. The leading malignancies that metastasize to heart are lung, breast and hematologic malignancies with the rates of $36-39 \%, 10-12 \%$ and $10-21 \%$, respectively. ${ }^{1}$ Prostate cancer is the most common malignancy in men, but nevertheless hardly metastasize to heart due to its low aggressiveness. ${ }^{2}$

The common mechanism of lung cancer to metastasize to the heart is by means of direct extension to epicardium or pericardium. ${ }^{2}$ Other possible pathways for various malignancies are lymphatic (most common) and hematogenous spread. Renal cell carcinoma is the leading cause for transvenous (caval) extension. Yet, lung cancer tends to use pulmonary veins to reach left atrium. ${ }^{3}$ Myocardial metastasis is rare and endocardial or intracavitary metastases are even rarer. The right chambers are affected mostly up to $80 \%$ and right atrium is the common site for metastases. ${ }^{4}$ 
Due to most cardiac metastases are indolent and course without cardiac specific symptoms, the diagnose often delay untill the advanced stages of the disease even at autopsy if not detected incidentally by imaging. ${ }^{1}$ If any symptom occurs, location and size of the tumor or invasion to surrounding organs may determine the clinical scenario. The involved chambers or valves may be obstructed, coronary flow may be altered due to mass effect, contraction abnormalities or arrhythmic interferences may occur ${ }^{5}$. Direct invasion of the tumor can result malignant pericardial effusion.

Echocardiography is the first line imaging modality to detect any obvious masses in any chambers, valves or apparent myocardial thickening. ${ }^{5,6}$ Transesophageal echocardiography (TEE) is superior than any other imaging modalities to reveal valvular mass lesions and its hemodynamical consequences. ${ }^{5}$ Computed tomography $(\mathrm{CT})$ and cardiac magnetic resonance $(\mathrm{CMR})$ are useful tools to detect, localize and characterize the

mass. CMR has the unique feature to reveal tissue characteristics which is of utmost importance in case of any myocardial mass. ${ }^{6}$ Contrast administration may aid in differentiating between thrombus and tumor. ${ }^{6}$

In conclusion, though cardiac metastases in lung cancer patients are expressed at the late stages of the disease, it is vital to detect any metastasis to adequately manage the patients as survival rate prolongs with the aid of novel therapies. Patients with known malignancy necessitates clinicians, echographers or imagers being more vigilant in case of unexpected myocardial thickening as it might resemble hypertrophic cardiomyopathy phenotype.

\section{CONFLICT OF INTEREST}

None.

\section{CONSENT}

Written consent was obtained from the patients.

\section{REFERENCES}

1. Bussani R, De-Giorgio F, Abbate A, et al. Cardiac metastases. J Clin Pathol 2007;60:27-34.

2. Hiremath R, K C A, Noronha GP, Naik SB, et al. Metastatic Cardiac Tumor from Bronchogenic Carcinoma via Pulmonary Vein: A Case Report. J Clin Diagn Res. 2013 Nov;7(11):2600-1.

3. Ferlay J, Shin HR, Bray F, et al. Estimates of worldwide burden of cancer in 2008: GLOBOCAN 2008. Int J Cancer 2010;127:2893-917.

4. Gadgeel SM, Kalemkerian GP. Lung cancer: Overview. In: Arenber D, Keshamouni V, Kalemkerian GP, editors. Lung Cancer Metastasis: Novel Biological Mechanisms and Impact on Clinical Practice. 1st ed. New York: Springer; 2009. p. 1-28.

5. Yarlagadda B, Paleti S, Annamalai SK. Multimodality Imaging to Characterize a Metastatic Cardiac Mass. Echocardiography. 2016 May;33(5):804-5.

6. Chiles C, Woodard PK, Gutierrez FR et al. Metastatic involvement of the heart and pericardium: CT and MR imaging. Radiographics 2001;21:439-49.

\section{Figure and Movie Legends}

Figure 1A-B. The apical 4ch (A) and parasternal short axis (B) transthoracic echocardiography images showed mass-like thickening of the septal myocardium (arrows) which nearly obscured the RV cavity. It also protruded toward LV cavity.

Figure 2A-C. The short axis T2 weighted (A) and transversal T1 weighted (B) CMR images showed significant myocardial thickening isointense to the normal myocardium. However, T1 mapping (C) clearly shows its heterogeneous signal intensity within the mass center.

Figure 3A-B. Late gadolinium images of short axis (A) and $4 \mathrm{ch}$ (B) images showed heterogeneously enhancing mass with its necrotic/hemorrhagic center (asterix).

Movie 1A-B. The apical 4ch (A) and parasternal short axis (B) transthoracic echocardiography images showed mass-like thickening of the septal myocardium. 
Movie 2A-B. The $3 \mathrm{ch}$ and $4 \mathrm{ch}$ cine images of CMR showed mass-like isointense myocardial thickening apparently obscuring both ventricle apices.

Movie 3. First-pass gadolinium perfusion study showed intense enhancement of the mass and the necrotic core.

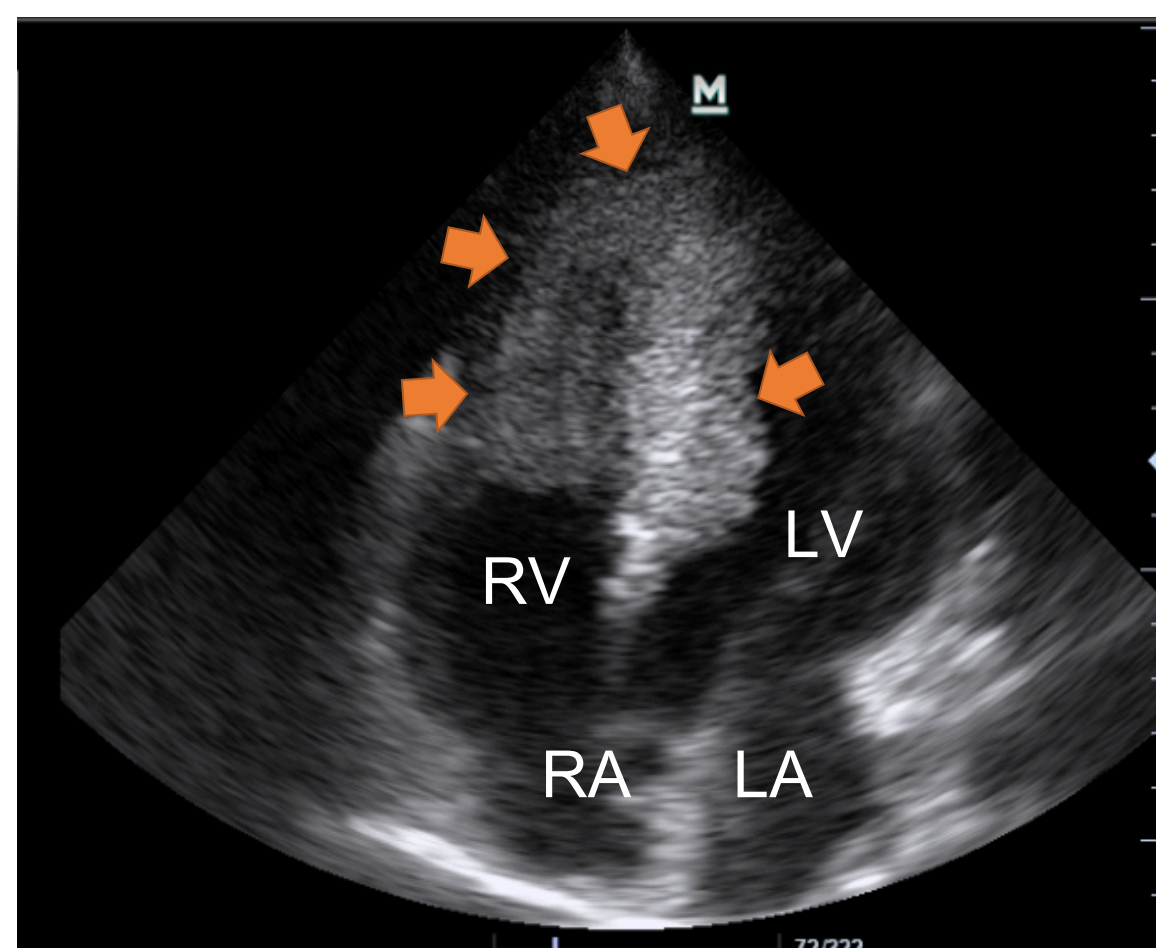




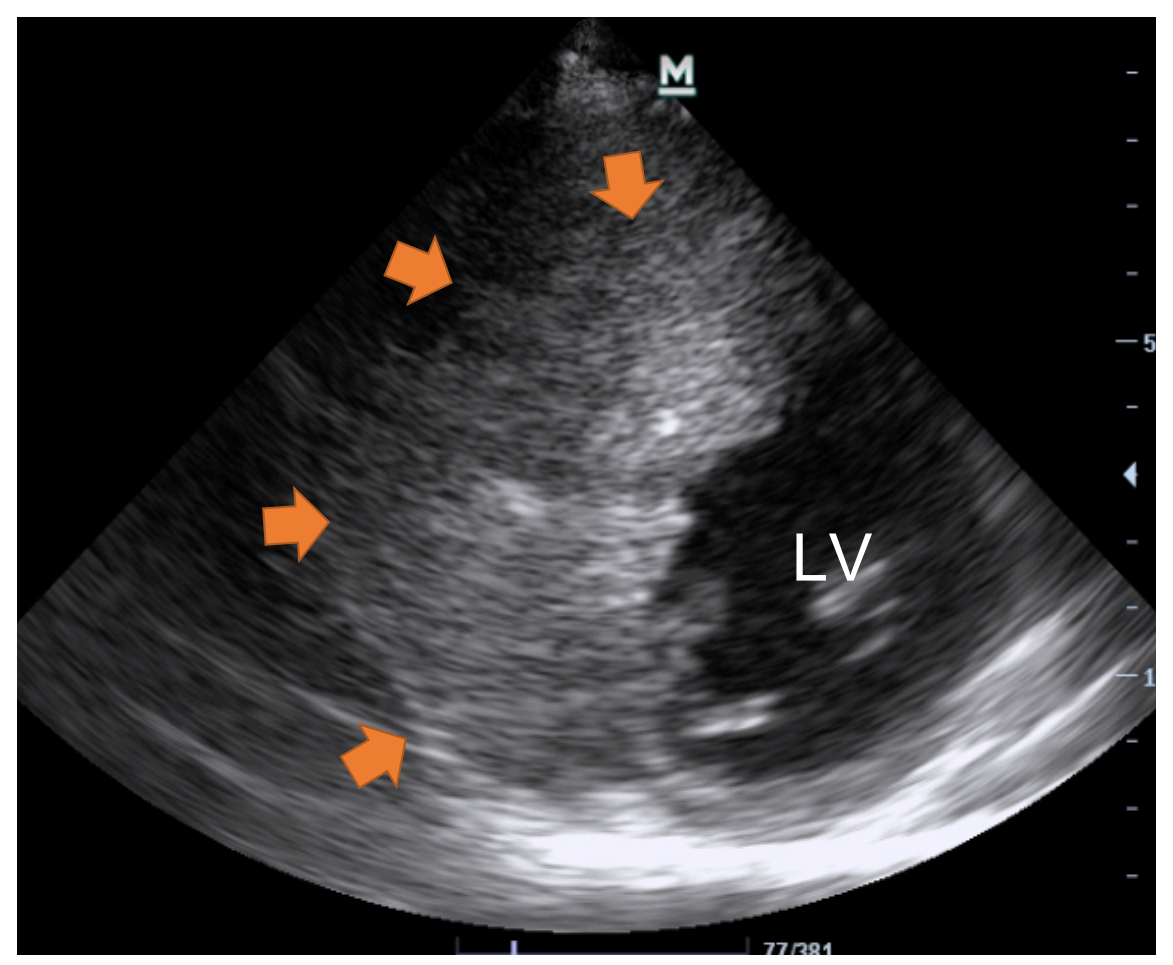




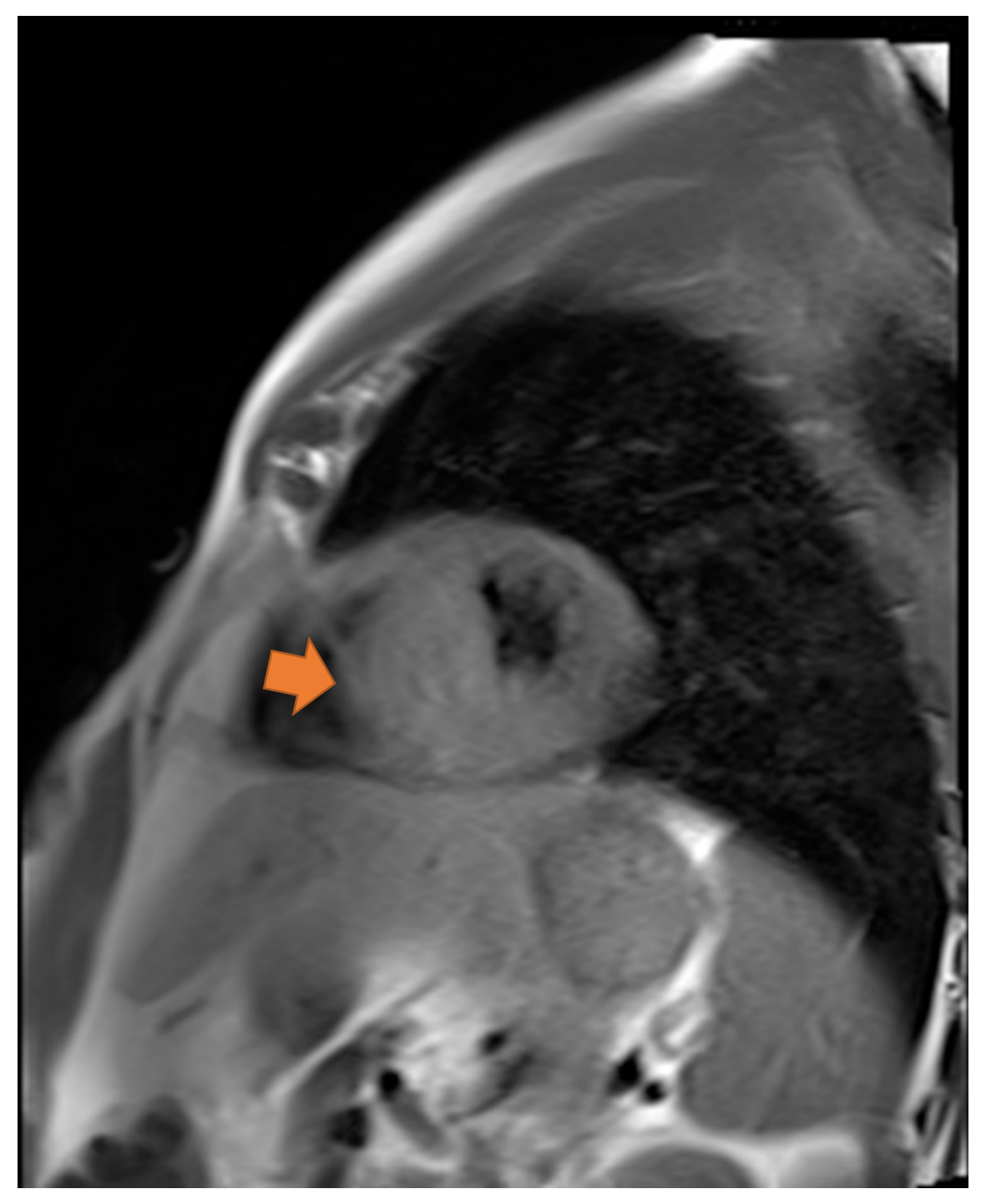



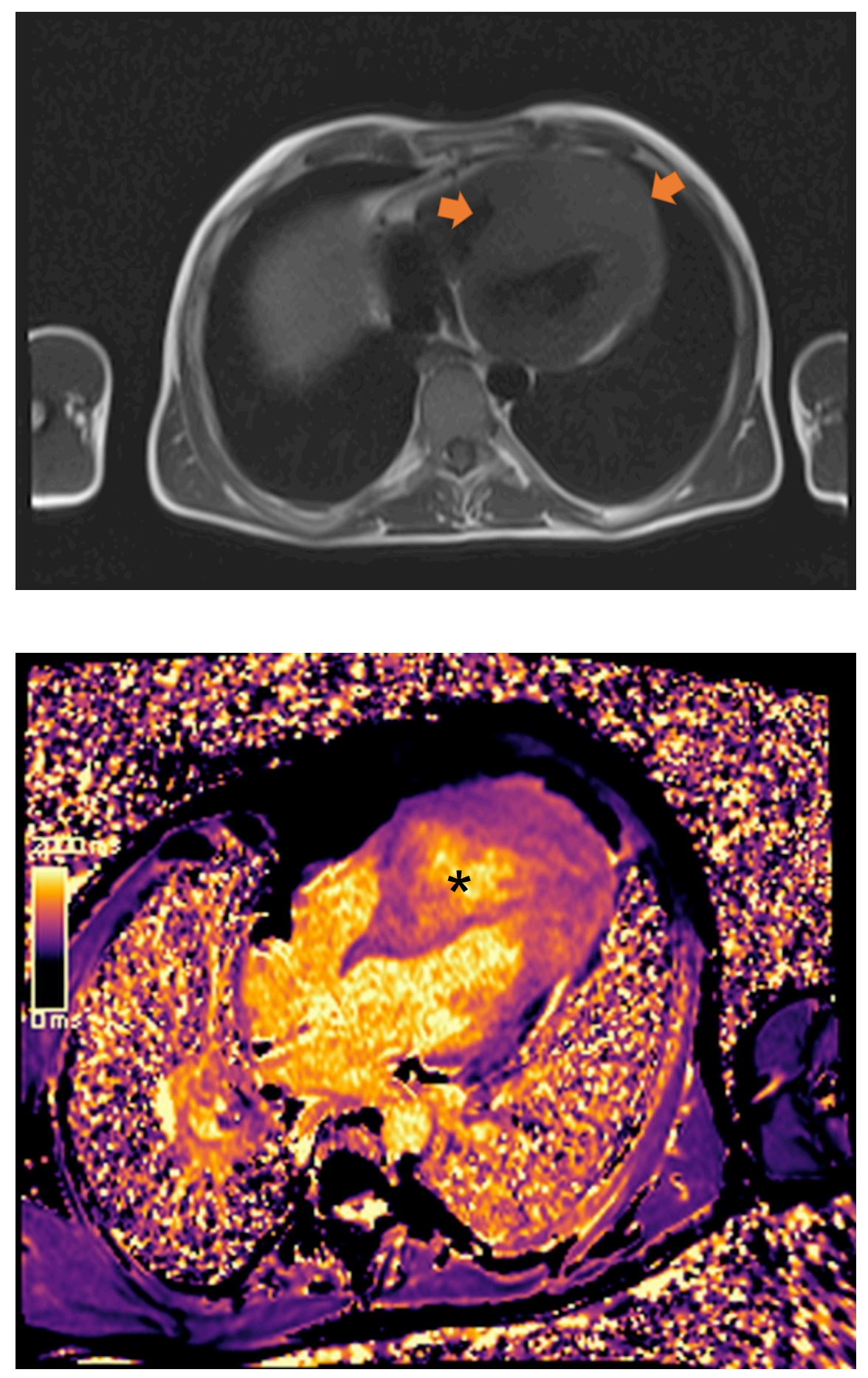


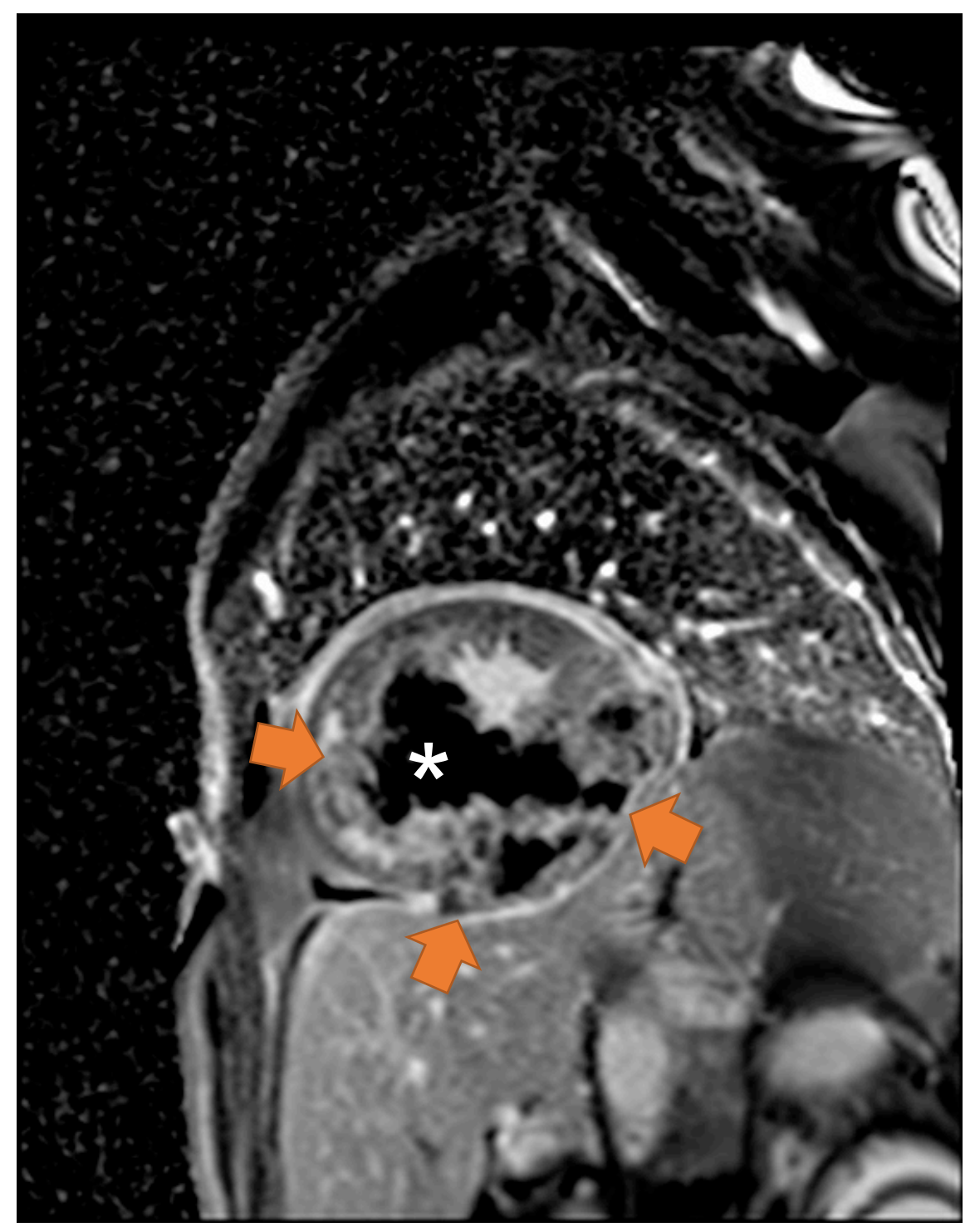




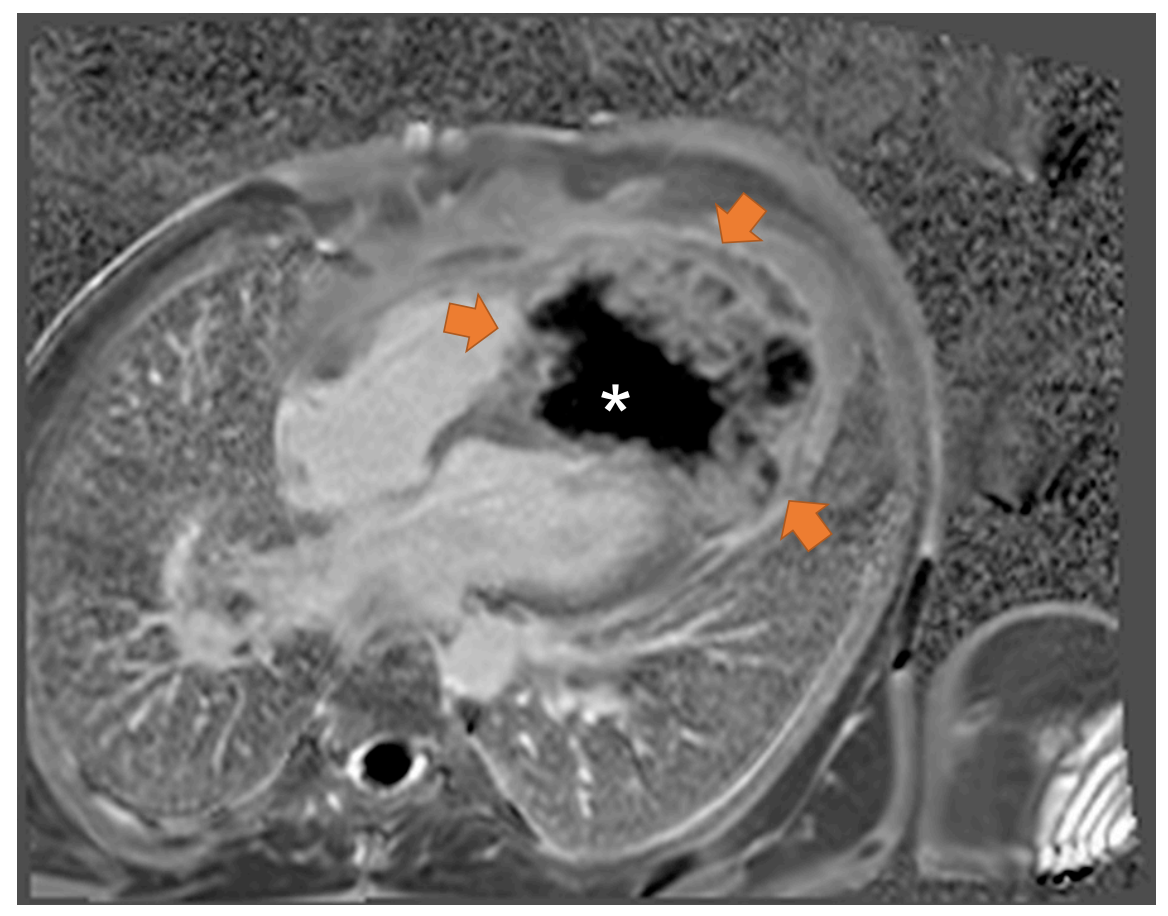

